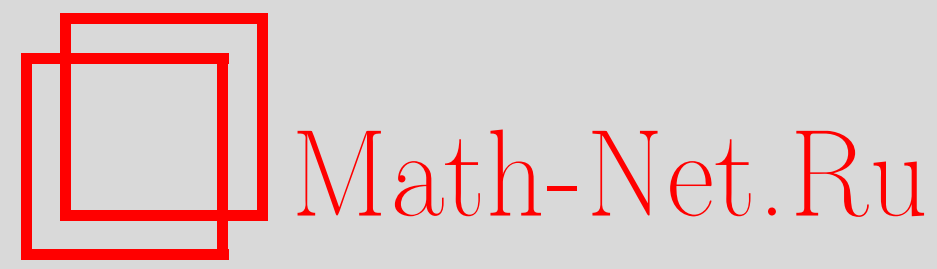

С. В. Пазизин, Б. В. Рязанов, Асимптотическая нормальность логарифма отношения правдоподобия одного типа $m$ зависимых случайных величин, Теория вероятн. и ее примен., 1998, том 43, выпуск 3, 476-489

DOI: https://doi.org/10.4213/tvp1555

Использование Общероссийского математического портала Math-Net.Ru подразумевает, что вы прочитали и согласны с пользовательским соглашением http://www . mathnet.ru/rus/agreement

Параметры загрузки:

IP : 3.85 .73 .92

26 апреля 2023 г., 16:18:58

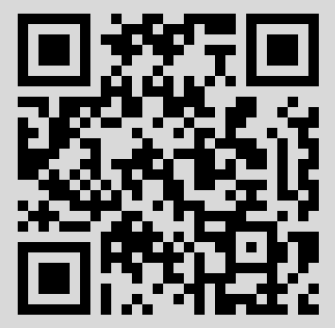


(c) 1998 г. ПАЗИЗИН С. В. ${ }^{*}$, РЯЗАНОВ Б. В. ${ }^{* *}$

\title{
АСИМПТОТИЧЕСКАЯ НОРМАЛЬНОСТЬ ЛОГАРИФМА ОТНОШЕНИЯ ПРАВДОПОДОБИЯ ОДНОГО ТИПА $m$-ЗАВИСИМЫХ СЛУЧАЙНЫХ ВЕЛИЧИН
}

\begin{abstract}
В работе показана асимптотическая нормальность логарифма отношения правдоподобия (ЛОП) в задаче проверки двух простых гипотез о распределении последовательности $m$-зависимых случайных величин по «откликам» на элементы этой последовательности.
\end{abstract}

Ключевые слова и фразы: $m$-зависимость, отношение правдоподобия, асимптотическая нормальность.

\section{1. Постановка задачи. Результат}

Пусть 1) $\mathfrak{L}_{\mathbb{X}}=\left\{\mathbb{X}, \mathfrak{B}_{\mathbb{X}}\right\}$ и $\mathfrak{U}_{\mathbb{Y}}=\left\{\mathbb{Y}, \mathfrak{B}_{\mathbb{Y}}\right\}-$ два измеримых пространства;

2) $X=\left\{X_{t}\right\}_{1}^{\infty}-$ стационарная в узком смысле последовательность $m$-зависимых случайных величин со значениями в $\mathbb{X}$, распределенная в соответствии с вероятностной мерой $Q \in\left\{Q_{1}, Q_{2}\right\}$, где $Q_{1}$ и $Q_{2}-$ две вероятностные меры на $\mathfrak{U}_{\mathbb{X}}^{\infty}$;

3) $Y=\left\{Y_{t}\right\}_{1}^{n}$ - серия случайных величин $Y_{t}=Y_{t}^{n}, t=1, \ldots, n$, со значениями в $\mathbb{Y}$, связанная с $X$ условным распределением:

$$
\mathbf{P}\left\{Y_{t} \in A_{t} ; t=1, \ldots, n \mid X\right\}=\prod_{t=1}^{n} \int_{A_{t}} p^{n}\left(y \mid X_{t}\right) d \mu(y),
$$

где $A_{t} \in \mathfrak{B}_{\mathbb{Y}}, t=1, \ldots, n$, а $\mathfrak{P}^{n}=\left\{p(y \mid x)=p^{n}(y \mid x) ; x \in \mathbb{X}\right\}-$ семейство плотностей (параметризованное элементами $x \in \mathbb{X}$ ) относительно некоторой общей доминирующей меры $\mu(y)$ на $\mathfrak{U}_{\mathbb{Y}}$. Причем, при любом фиксированном $y \in \mathbb{Y}, p(y \mid x)$ - измеримая относительно $\mathfrak{B}_{\mathbb{X}}$ функция от $x$.

Ставится задача - по наблюдаемой серии $Y$ различить две гипотезы $H_{1}\left(Q=Q_{1}\right)$ и $H_{2}\left(Q=Q_{2}\right)$ о распределении последовательности $X$.

*Академия ФСБ РФ, Мичуринский пр., 62, 117602 Москва, Россия.

** Фннансовая компания «Интраст», ул. Вавилова, 69-75, 117846 Москва, Россия. 
Меры $Q_{1}, Q_{2}$ и соотношение $(1)$ индуцируют на $\mathfrak{U}_{\mathbb{Y}}^{n}$ соответствуюшие меры $P_{1}^{n}$ и $P_{2}^{n}$. Рассмотрим логарифм отношения правдоподобия (ЛОП)

$$
L_{n}=\ln \frac{d P_{2}^{n}(Y)}{d P_{1}^{n}(Y)}
$$

при $n \rightarrow \infty$ и $\delta \rightarrow 0$, где

$$
\begin{aligned}
\delta^{2} & =\delta_{n}^{2}=\int_{\mathbb{Y}^{*}} \frac{\left(p_{2}(y)-p_{1}(y)\right)^{2}}{p_{1}(y)} d \mu(y) \\
\left(\mathbb{Y}^{*}\right. & \left.=\left\{y \in \mathbb{Y}: p_{1}^{n}(y)>0\right\} \bigcup\left\{y \in \mathbb{Y}: p_{2}^{n}(y)>0\right\}\right)
\end{aligned}
$$

- расстояние $\chi^{2}$ между плотностями $p_{1}(y)=p_{1}^{n}(y)$ и $p_{2}(y)=p_{2}^{n}(y)$ распределений случайной величины $Y_{1}$, отвечающими гипотезам $H_{1}$ и $H_{2}$ соответственно.

В задачах статистики $m$-зависимые случайные величины возникают в основном при рассмотрении последовательностей вида

$$
\eta_{t}=f\left(\xi_{t}, \ldots, \xi_{t+m}\right), \quad t=1,2, \ldots,
$$

где $\xi_{1}, \xi_{2}, \ldots$ - независимые случайные величины, $f$ - некоторая измеримая функция от $m+1$ переменной (см. [1]). Предельное поведение сумм $m$-зависимых случайных величин изучалось в работах [2]-[4] и др. Более общий случай слабо зависимых стационарно связанных случайных величин рассматрұгвался в [1], [5]. Предельные теоремы для сумм слабо зависимых величин в схеме серий содержатся в [6]. В ряде работ изучалось поведение $U$-статистик от $m$-зависимых случайных величин (cм. [7]).

Предельное поведение ЛОП $m$-зависимых случайных величин исследовалось в работах, связанных с изучением характеристик датчиков случайньтх чисел (см. [8]), а также с построением асимптотически оптимальных критериев в некоторых задачах статистической радиотехники (см. [9]). Изучению процессов отношения правдоподобия для различных моделей посвящено большое количество работ (см. монографии [10]-[12]). Возможность применения в данном случае общих результатов (см. [13]) затрудняется сложностью получения оценок условных вероятностей вида: $\mathbf{P}\left\{Y_{t+1}=y \mid Y_{t}, Y_{t-1}, \ldots, Y_{1}\right\}, t \in \mathbf{N}$, и тем, что рассматривается не только контигуальный случай $\left(\delta^{2} n=\mathrm{const}\right)$, но и случай расходящихся гипотез $\left(\delta^{2} n \rightarrow \infty\right)$. По этим же причинам в работе не использовались мартингальные методы ([14]). Доказательство асимптотической нормальности ЛОП проводится путем многошаговой редукции, основанной на последовательном применении метода С. Н. Бернштейна ([15]).

Теорема. Пусть $n \rightarrow \infty, \delta \rightarrow 0$,

$$
\frac{p\left(y \mid x_{1}\right)}{p\left(y \mid x_{2}\right)}-1=O(\delta)
$$


равномерно по $y \in \mathbb{Y}$ и $x_{1}, x_{2} \in \mathbb{X}$ и выполняотся соотночения:

$$
m=o(n), \quad \delta m^{12} \rightarrow 0
$$

uлu

$$
\delta^{2} n=\text { const }(>0), \quad \delta m^{6} \rightarrow 0 .
$$

Тогда, npu аuпотезе $H_{\varepsilon}(\varepsilon=1,2)$ :

1) распределение случайной величины

$$
\widetilde{L}_{n}=\frac{L_{n}-\mathbf{E}_{\varepsilon} L_{n}}{\sqrt{\mathbf{D}_{\varepsilon} L_{n}}}
$$

слабо сходится $к$ распределению стандартного нормального закона;

2) среднее и дисперсия статистики $L_{n}$ ведут себя следуюшим образом:

$$
\mathbf{E}_{\varepsilon} L_{n} \sim(-1)^{\varepsilon} \frac{\delta^{2} n}{2}, \quad \mathbf{D}_{\varepsilon} L_{n} \sim \delta^{2} n .
$$

Следствие. В условиях теоремы статистика

$$
S_{n}=\sum_{t=1}^{n}\left(\frac{p_{2}\left(Y_{t}\right)}{p_{1}\left(Y_{t}\right)}-1\right)
$$

является асимптотически достаточной в задаче проверки аипотез $H_{1} u H_{2}$ no $Y$.

Для доказательства данного следствия достаточно заметить, что в условиях теоремы при гипотезе $H_{\varepsilon}(\varepsilon=1,2)$ распределение статистики

$$
\tilde{S}_{n}=\frac{1}{\delta \sqrt{n}} \sum_{t=1}^{n}\left(\frac{p_{2}\left(Y_{t}\right)}{p_{1}\left(Y_{t}\right)}-1+\delta^{2}(1-\varepsilon)\right)
$$

слабо сходится к распределению стандартного нормального закона. Последнее является попутным результатом доказательства теоремы.

3 а м е т а н и е. Если $X$ - не стационарная последовательность, $p_{t \varepsilon}(y)=p_{t \varepsilon}^{n}(y)$ - плотность распределения $Y_{t}$ при гипотезе $H_{\varepsilon}(\varepsilon=1,2)$ и для всех $t=1, \ldots, n$

$$
\begin{gathered}
\delta^{2} \sim \delta_{n}^{2}(t)=\int_{\mathbb{Y}_{i}^{*}} \frac{\left(p_{t 2}(y)-p_{t 1}(y)\right)^{2}}{p_{t 1}(y)} d \mu(y) \\
\left(\mathbb{Y}_{t}^{*}=\left\{y \in \mathbb{Y}: p_{t 1}(y)>0\right\} \bigcup\left\{y \in \mathbb{Y}: p_{t 2}(y)>0\right\}\right),
\end{gathered}
$$

т.е. расстояние $\chi^{2}$ между плотностями $p_{t 1}(y)$ и $p_{t 2}(y)$ в асимптотике не зависит от $t$, то теорема сохраняет свою силу, что нетрудно увидеть из доказательства. 
Заметим также, что в условиях теоремы расстояние $\chi^{2}$ при $n \rightarrow \infty$ ведет себя аналогично расстоянию Хеллингера:

$$
\delta^{2} \sim 4 \sum_{t=1}^{n} \int_{\mathbb{Y}}\left(\sqrt{p_{t 1}\left(y_{t}\right)}-\sqrt{p_{t 2}\left(y_{t}\right)}\right)^{2} d \mu(y)
$$

и в независимом случае при $\delta^{2} n=$ const $(>0)$ асимптотическая нормальность ЛОП вытекает из теоремы 2 работы [16].

\section{2. Доказательство теоремы}

Прежде всего отметим, что по условию (4) носители распределений из семейства $\mathfrak{P}$ совпадают с $\mathbb{Y}^{*}$ (далее будем считать, что $\mathbb{Y}^{*}=\mathbb{Y}$ ). Следовательно, меры $P_{1}^{n}$ и $P_{2}^{n}$ абсолютно непрерывны друг относительно друга для любого фиксированного $n \in \mathbf{N}$, и запись (2) является корректной в условиях теоремы.

Мы проведем доказательство только для гипотезы $H_{1}$. Для альтернативы $\mathrm{H}_{2}$ доказательство в общем случае проводится аналогично, а в случае $\delta^{2} n=$ const $(>0)$ вытекает из контигуальности гипотез $H_{1}$ й $H_{2}$. Индекс «1» (номер гипотезы $H_{1}$ ) при знаках математического ожидания и дисперсии далее будем опускать.

2.1. Оценим среднее и дисперсию $L_{n}$ при $\delta n \rightarrow 0$. Из представления (1) имеем:

$$
p_{\varepsilon}(\mathbf{y})=\frac{d P_{\varepsilon}^{n}(\mathbf{y})}{d \mu}=\int_{\mathbb{X}^{n}} \prod_{t=1}^{n} p\left(y_{t} \mid x_{t}\right) d Q_{\varepsilon}
$$

$\left(\mathbf{y}=\left(y_{1}, \ldots, y_{n}\right), d \mu=d \mu\left(y_{1}\right) \times \cdots \times d \mu\left(y_{n}\right), \varepsilon=1,2\right)$. Отсюда, согласно $(4)$,

$$
\frac{p_{\varepsilon}(\mathbf{y})}{\prod_{t=1}^{n} p_{1}\left(y_{t}\right)}=\int_{\mathbb{X}^{n}} \prod_{t=1}^{n}\left(1+\frac{p\left(y_{t} \mid x_{t}\right)}{p_{1}\left(y_{t}\right)}-1\right) d Q_{\varepsilon}=\prod_{t=1}^{n}(1+O(\delta)) \sim 1,
$$

$\varepsilon=1,2$. Это позволяет, воспользовавшись разложением логарифма по формуле Тейлора, получить следующее:

$$
\begin{aligned}
\mathbf{E} L_{n} & =\mathbf{E}\left\{\left(\frac{p_{2}(Y)}{p_{1}(Y)}-1\right)-\frac{1}{2}\left(\frac{p_{2}(Y)}{p_{1}(Y)}-1\right)^{2}(1+o(1))\right\} \\
& \sim-\frac{1}{2} \int_{\mathbb{Y}^{n}} \frac{\left(p_{2}(\mathbf{y})-p_{1}(\mathbf{y})\right)^{2}}{p_{1}(\mathbf{y})} d \mu \\
\mathbf{E} L_{n}^{2} & =\mathbf{E}\left\{\left(\frac{p_{2}(Y)}{p_{1}(Y)}-1\right)(1+o(1))\right\}^{2} \sim \int_{\mathbb{Y}^{n}} \frac{\left(p_{2}(\mathbf{y})-p_{1}(\mathbf{y})\right)^{2}}{p_{1}(\mathbf{y})} d \mu .
\end{aligned}
$$


Оценим величину

$$
\begin{aligned}
\int_{\mathbb{Y}^{n}} & \frac{\left(p_{2}(\mathrm{y})-p_{1}(\mathrm{y})\right)^{2}}{p_{1}(\mathrm{y})} d \mu \\
\sim & \int_{\mathbb{Y}^{n}}\left(\frac{p_{2}\left(y_{1}, \ldots, y_{n}\right)-p_{1}\left(y_{1}, \ldots, y_{n}\right)}{p_{1}\left(y_{1}\right) \times \cdots \times p_{1}\left(y_{n}\right)}\right)^{2} p_{1}\left(y_{1}\right) \times \cdots \times p_{1}\left(y_{n}\right) d \mu \\
= & \int_{\mathbb{Y}^{n}}\left(\int_{\mathbb{X}^{n}} \prod_{t=1}^{n}\left(1+\frac{p\left(y_{t} \mid x_{t}\right)}{p_{1}\left(y_{t}\right)}-1\right) d Q_{2}\right. \\
= & \left.\quad-\int_{\mathbb{X}^{n}} \prod_{t=1}^{n}\left(1+\frac{p\left(y_{t} \mid x_{t}\right)}{p_{1}\left(y_{t}\right)}-1\right) d Q_{1}\right)^{2} p_{1}\left(y_{1}\right) \times \cdots \times p_{1}\left(y_{n}\right) d \mu \\
& \left.\left.\quad-\int_{\mathbb{X}^{n}}^{n} \prod_{j=1}^{n}\left(\frac{p\left(y_{t_{j}} \mid x_{t_{j}}\right)}{p_{1}\left(y_{t_{j}}\right)}-1\right) d Q_{1}\right)\right)^{2} p_{1}\left(y_{1}\right) \times \cdots \times p_{1}\left(y_{n}\right) d \mu \\
= & \int_{\mathbb{Y}^{n}} \sum_{r=1}^{n} \sum_{1 \leqslant t_{1}<\cdots<t_{r} \leqslant n}\left(\int_{\mathbb{X}^{n}} \prod_{j=1}^{r}\left(\frac{p\left(y_{t_{j}} \mid x_{t_{j}}\right)}{p_{1}\left(y_{t_{j}}\right)}-1\right) d Q_{2}\right. \\
& \left.\quad-\int_{\mathbb{X}^{n}} \prod_{j=1}^{r}\left(\frac{p\left(y_{t_{j}} \mid x_{t_{j}}\right)}{p_{1}\left(y_{t_{j}}\right)}-1\right) d Q_{1}\right)^{2} p_{1}\left(y_{1}\right) \times \cdots \times p_{1}\left(y_{n}\right) d \mu .
\end{aligned}
$$

Для доказательства последнего равенства достаточно поменять порядок интегрирования соответствующих слагаемых и воспользоваться тем, что для всех $t=1, \ldots, n$ и любого $x \in \mathbb{X}$

$$
\mathbf{E}\left(\frac{p\left(Y_{t} \mid x\right)}{p_{1}\left(Y_{t}\right)}-1\right)=\int_{\mathbb{Y}}\left(\frac{p(y \mid x)}{p_{1}(y)}-1\right) p_{1}(y) d \mu=0 .
$$

Далее, согласно (4), получаем:

$$
\begin{aligned}
& \int_{\mathbb{Y}^{n}} \frac{\left(p_{2}(\mathrm{y})-p_{1}(\mathrm{y})\right)^{2}}{p_{1}(\mathrm{y})} d \mu \\
& \quad \sim \int_{\mathbb{Y}^{n}} \sum_{t=1}^{n}\left(\frac{p_{2}\left(y_{t}\right)-p_{1}\left(y_{t}\right)}{p_{1}\left(y_{t}\right)}\right)^{2} p_{1}\left(y_{t}\right) d \mu+O\left(\delta^{2} \sum_{r=2}^{n} n^{r} \delta^{2(r-1)}\right) \\
& \quad \sim \delta^{2} n+O\left(\delta^{2}\left(n^{2} \delta^{2}\right)\left(\sum_{r=0}^{n-2} n^{r} \delta^{2 r}\right)\right) \sim \delta^{2} n .
\end{aligned}
$$

Отсюда и из (9) и (10) вытекает:

$$
\begin{gathered}
\mathbf{E} L_{n} \sim-\frac{1}{2} \delta^{2} n, \\
\mathbf{D} L_{n}^{2}=\mathbf{E} L_{n}^{2}-\left(\mathbf{E} L_{n}\right)^{2} \sim \delta^{2} n .
\end{gathered}
$$


2.2. Покажем асимптотическую нормальность $\widetilde{L}_{n}$ при $\delta n \rightarrow 0$. Положим

$$
S_{n}=\sum_{t=1}^{n} \zeta_{t}
$$

где

$$
\zeta_{t}=\frac{p_{2}\left(Y_{t}\right)}{p_{1}\left(Y_{t}\right)}-1, \quad t=1, \ldots, n \text {. }
$$

Легко показать, что $\mathrm{E} S_{n}=0$, a

$$
\mathbf{E} S_{n}^{2}=\sum_{t=1}^{n} \mathbf{E} \zeta_{t}^{2}+\sum_{t \neq k} \mathbf{E} \zeta_{t} \zeta_{k}=\delta^{2} n+O\left(\delta^{4} m n\right) \sim \delta^{2} n
$$

так как, согласно представлению (8) и условию (4), для любых $t \neq k$

$$
\begin{aligned}
\mathbf{E} \zeta_{t} \zeta_{k}= & \int_{\mathbb{Y}^{n}}\left(\frac{p_{2}\left(y_{t}\right)}{p_{1}\left(y_{t}\right)}-1\right)\left(\frac{p_{2}\left(y_{k}\right)}{p_{1}\left(y_{k}\right)}-1\right) \int_{\mathbb{X}^{n}}\left(p\left(y_{t} \mid x_{t}\right)-p_{1}\left(y_{t}\right)+p_{1}\left(y_{t}\right)\right) \\
& \times\left(p\left(y_{k} \mid x_{k}\right)-p_{1}\left(y_{k}\right)+p_{1}\left(y_{k}\right)\right) d Q_{1} d \mu \\
= & \int_{\mathbb{Y}^{n}}\left(\frac{p_{2}\left(y_{t}\right)}{p_{1}\left(y_{t}\right)}-1\right)\left(\frac{p_{2}\left(y_{k}\right)}{p_{1}\left(y_{k}\right)}-1\right) \int_{\mathbb{X}^{n}}\left(p\left(y_{t} \mid x_{t}\right)-p_{1}\left(y_{t}\right)\right) \\
& \times\left(p\left(y_{k} \mid x_{k}\right)-p_{1}\left(y_{k}\right)\right) d Q_{1} d \mu=O\left(\delta^{4}\right) .
\end{aligned}
$$

Рассмотрим, при $\delta n \rightarrow 0$, центрированную статистику

$$
\bar{L}_{n}=L_{n}+\frac{\delta^{2} n}{2}
$$

Из соотношений (11), (12) и (14) следует, что

$$
\begin{aligned}
\mathbf{E}\left(L_{n}-S_{n}\right)^{2}= & \mathbf{E} L_{n}^{2}-2 \mathbf{E} L_{n} S_{n}+\mathbf{E} S_{n}^{2}=\mathbf{E} L_{n}^{2}+\mathbf{E} S_{n}^{2} \\
& -2 \mathbf{E}\left(\frac{p_{2}(Y)}{p_{1}(Y)}-1\right) \sum_{t=1}^{n}\left(\frac{p_{2}\left(Y_{t}\right)}{p_{1}\left(Y_{t}\right)}-1\right)+o\left(2 \delta^{2} n\right) \\
= & 2 \delta^{2} n+o\left(\delta^{2} n\right)-2 \sum_{t=1}^{n} \int_{\mathbb{Y}^{n}}\left(\frac{p_{2}^{2}\left(y_{t}\right)}{p_{1}\left(y_{t}\right)}-p_{2}\left(y_{t}\right)\right) d \mu \\
= & 2 \delta^{2} n+o\left(\delta^{2} n\right)-2 \delta^{2} n=o\left(\delta^{2} n\right) .
\end{aligned}
$$

Отсюда и из (14) имеем: $\mathbf{D}\left(\bar{L}_{n}-S_{n}\right)=o\left(\mathbf{D} S_{n}\right)$. Следовательно, предельное распределение $\bar{L}_{n}$ совпадает с предельным распределением $S_{n}$, и дело, таким образом, сводится к исследованию асимптотического поведения статистики $S_{n}$.

Для доказательства асимптотической нормальности статистики $\widetilde{S}_{n}=S_{n} / \delta \sqrt{n}$ воспользуемся методом С. Н. Бернштейна, разработанным для распространения пентральной предельной теоремы на слабозависимые случайные величины ([12]). 
Пусть $h=[\sqrt{n m}]$. Так как $m=o(n)$, то $m=o(h)$, а $h=o(n)$. Разобьем сумму $S_{n}$ на участки

$$
\begin{aligned}
& \zeta_{1}^{\prime}=\zeta_{1}+\cdots+\zeta_{h}, \quad \zeta_{1}^{\prime \prime}=\zeta_{h+1}+\cdots+\zeta_{h+m}, \\
& \zeta_{2}^{\prime}=\zeta_{h+m+1}+\cdots+\zeta_{2 h+m}, \quad \zeta_{2}^{\prime \prime}=\zeta_{2 h+m+1}+\cdots+\zeta_{2 h+2 m} \text {, } \\
& \text {.............. } \quad \text {.............. }
\end{aligned}
$$

длины $h$ и $m$ соответственно. Таким образом, сумма $S_{n}$ распадается на две части $S^{\prime}=\zeta_{1}^{\prime}+\cdots+\zeta_{M}^{\prime}$ и $S^{\prime \prime}=\zeta_{1}^{\prime \prime}+\cdots+\zeta_{M}^{\prime \prime}$, каждая из которых представляет собой сумму независимых случайных величин. При этом в силу выбора $h$ дисперсия $S^{\prime \prime}$ по порядку меньше дисперсии $S^{\prime}$, и, значит, предельное распределение суммы $S_{n}$ совпадает с предельным распределением суммы $S^{\prime}$.

Для статистики $S^{\prime}$ выполняется обеспечивающее ее асимптотическую нормальность условие Ляпунова

$$
M \mathrm{E}\left(\zeta^{\prime}\right)^{4}=o\left(\left(\mathrm{D} S^{\prime}\right)^{2}\right)
$$

Чтобы в этом убедиться, покажем, что

$$
\mathrm{E} S_{n}^{4}=O\left(\left(\delta^{2} n\right)^{2}\right),
$$

для чего представим четвертый момент суммы (13) в виде

$$
\mathrm{E} S_{n}^{4}=\Sigma_{0}+4 \Sigma_{1}+3 \Sigma_{2}+3 \Sigma_{3}+\Sigma_{4},
$$

где

$$
\begin{aligned}
& \Sigma_{0}=\Sigma \mathbf{E} \zeta_{t}^{4}, \quad \Sigma_{1}=\Sigma \mathbf{E} \zeta_{t_{1}}^{3} \zeta_{t_{2}}, \quad \Sigma_{2}=\Sigma \mathbf{E} \zeta_{t_{1}}^{2} \zeta_{t_{2}}^{2}, \\
& \Sigma_{3}=\Sigma \mathbf{E} \zeta_{t_{1}}^{2} \zeta_{t_{2}} \zeta_{t_{3}}, \quad \Sigma_{4}=\Sigma \mathbf{E} \zeta_{t_{1}} \zeta_{t_{2}} \zeta_{t_{3}} \zeta_{t_{4}}
\end{aligned}
$$

и суммирование в $\Sigma_{i}$ ведется по попарно неравным индексам. Согласно (4),

$$
\Sigma_{0}=O\left(\delta^{4} n^{2}\right), \quad \Sigma_{1}=O\left(\delta^{4} n^{2}\right), \quad \Sigma_{2}=O\left(\delta^{4} n^{2}\right) .
$$

Применив прием, использованный при доказательстве (14), получаем:

$$
\Sigma_{3}=O\left(\delta^{6} n^{2} m\right), \quad \Sigma_{4}=O\left(\delta^{8} n^{2} m^{2}\right) .
$$

Следовательно, (16) выполнено, и на основании того, что $M \sim n / h \sim$ $\sqrt{n / m}, h=\sqrt{n m}, D S^{\prime} \sim D S_{n} \sim \delta^{2} n$, получаем соотношения

$$
M \mathrm{E}\left(\zeta^{\prime}\right)^{4}=M \mathrm{E}\left(S_{h}\right)^{4} \sim M \delta^{4} h^{2} \sim \delta^{4} n m \sqrt{\frac{n}{m}}=o\left(\delta^{4} n^{2}\right),
$$

доказываюшие (15). Асимптотическую нормальность статистики $\widetilde{S}_{n}$ несложно показать и в более общем случае, воспользовавшись известными результатами для схем серий слабозависимых случайных величин. 
В данном случае мы предпочли воспользоваться методом С. Н. Бернштейна, тақ кақ полученные при этом соотношения используются в дальнейшем, когда идея этого метода применяется для сведения случая $\delta n \geqslant C>0$ к случаю $\delta n \rightarrow 0$ при исследовании ЛОП. (Здесь и далее символом $C$ обозначаются неуточняемые константы.)

2.3. Опишем процедуру «дробления» $L_{n}$ в случае $\delta n \geqslant C>0$ и $\delta m^{12} \rightarrow 0$.

1) Лемма 1. Пусть $\Delta>0, \Delta \rightarrow 0, n \rightarrow \infty$. Тогда существует неотричательная челочисленная функчия $r^{\prime}=r^{\prime}(\Delta, n)$, для которой

$$
\Delta^{r^{\prime}} n \rightarrow \infty, \quad \Delta^{r^{\prime}+2} n \rightarrow 0 .
$$

Д ок аз а те льс т в о. Положим $a=\ln n, b=\ln \Delta^{-1}$. Очевидно, что соотношения (17) эквивалентны соотношениям

$$
a-r^{\prime} b \rightarrow \infty, \quad\left(r^{\prime}+2\right) b-a \rightarrow \infty,
$$

и при этом $a \rightarrow \infty, b \rightarrow \infty$.

Функцию $r^{\prime}$ строим следующим образом. Для данных $a$ и $b$ выберем такое неотрицательное целое $i$, что $i b<a \leqslant(i+1) b$. В случае $i=0$ полагаем $r^{\prime}=0$. В случае $i \geqslant 1$ полагаем

$$
r^{\prime}= \begin{cases}i-1, & \text { если } i b<a \leqslant\left(i+\frac{1}{2}\right) b, \\ i, & \text { если }\left(i+\frac{1}{2}\right) b<a \leqslant(i+1) b .\end{cases}
$$

Легко видеть, что в случае $r^{\prime}=0$

$$
a-r^{\prime} b=a, \quad\left(r^{\prime}+2\right) b-a=2 b-a \geqslant b,
$$

а в случае $r^{\prime} \geqslant 1$

$$
a-r^{\prime} b \geqslant \frac{b}{2}, \quad\left(r^{\prime}+2\right) b-a \geqslant \frac{b}{2}
$$

откуда следует (18). Лемма 1 доказана. функщию

Положим $d=\left[\delta^{-1 / 3}\right], \Delta=d^{-1}$ и на основе леммы 1 определим

$$
r=r(\Delta, n)= \begin{cases}0, & \text { если } r^{\prime}=0, \\ r^{\prime}-1, & \text { если } r^{\prime} \geqslant 1 .\end{cases}
$$

Далее, положим $n_{0}=n, n_{i}=\left[\Delta n_{i-1}\right]-m, i=1, \ldots, r$.

Лемма 2. $B$ случае $\delta n \geqslant C>0(n \rightarrow \infty, \delta \rightarrow 0)$ параметры $d, m, r, \delta, \Delta u n_{i}(i=0, \ldots, r)$ ведут себя следующим образом:

$$
\Delta \rightarrow 0, \quad \Delta \sim \delta^{1 / 3}, \quad r \geqslant 1 \text {; }
$$


2)

$$
\Delta^{r+1} n \rightarrow \infty, \quad \Delta^{r+3} n \rightarrow 0,
$$

$$
n_{i} \sim \Delta^{i} n, \quad n_{i} \rightarrow \infty \text { равномерно по } i, 0 \leqslant i \leqslant r ;
$$

3) $\quad \gamma=\delta m d \rightarrow 0, \quad m^{4}=o(d), \quad m^{2}=o\left(\Delta^{r+1} n\right)$.

Д о к а 3 а т е лг с с т в о. Соотношения (21) и (22) являются прямым следствием леммы 1 , определения (20) и условия $\Delta^{3} n \sim \delta n \geqslant C>0$. Первое и второе соотношения в (24) вытекают из условий теоремы и определения $d$ и $\gamma$. Последняя оценка в (24) вытекает из второй и неравенства $\Delta^{r+1} n \geqslant \Delta^{-1 / 2}=\sqrt{d}$, которое является следствием неравенств (19). Для доказательства (23) применим последовательно неравенства $\Delta n_{j-1}-(m+1) \leqslant n_{j} \leqslant \Delta n_{j-1}-m, 1 \leqslant j \leqslant i$, в результате чего получим соотношения

$$
\Delta^{i} n-(m+1) \frac{1-\Delta^{i}}{1-\Delta} \leqslant n_{i} \leqslant \Delta^{i} n-m \frac{1-\Delta^{i}}{1-\Delta}, \quad 1 \leqslant i \leqslant r
$$

из которых следует, что $\left|n_{i}-\Delta^{i} n\right| \leqslant C m$. Но, согласно (24), $m=o\left(\Delta^{i} n\right)$ равномерно по $i(1 \leqslant i \leqslant r)$, и, значит $n_{i}=\Delta^{i} n\{1+o(1)\}$. Лемма 2 доказана.

2) Приступим к непосредственному исследованию исходной статистики

$$
\begin{aligned}
\mathfrak{M}_{0}=L_{n}= & \ln \left(\int_{\mathbb{X}^{n}} \exp \sum_{t=1}^{n} \ln \frac{p\left(Y_{t} \mid x_{t}\right)}{p_{1}\left(Y_{t}\right)} d Q_{2}\right) \\
& -\ln \left(\int_{\mathbb{X}^{n}} \exp \sum_{t=1}^{n} \ln \frac{p\left(Y_{t} \mid x_{t}\right)}{p_{1}\left(Y_{t}\right)} d Q_{1}\right) .
\end{aligned}
$$

Разобьем $\mathscr{M}_{0}=\sum_{t=1}^{n} \ln \left(p\left(Y_{t} \mid x_{t}\right) / p_{1}\left(Y_{t}\right)\right)$ на две части:

$$
\mathscr{M}_{0}=\sum_{j=0}^{d} \mathscr{M}_{1 j}=\mathscr{N}_{1}+\mathscr{M}_{10},
$$

объединив в основную часть $\mathscr{N}_{1}$ независимые слагаемые

$$
\mathscr{M}_{1 j}=\sum_{t=(j-1) h+1}^{(j-1) h+n_{1}} \xi_{t}, \quad j=1, \ldots, d,
$$

где

$$
\xi_{t}=\ln \frac{p\left(Y_{t} \mid x_{t}\right)}{p_{1}\left(Y_{t}\right)} \quad(t=1, \ldots, n), \dot{h}=h_{1}=n_{1}+m=[\Delta n] .
$$

В соответствии с этим разбиением представим статистику $\mathfrak{M}_{0}$ в виде

$$
\mathfrak{M}_{0}=\sum_{j=0}^{d} \mathfrak{M}_{1 j}=\mathfrak{N}_{1}+\mathfrak{M}_{10},
$$


где

$$
\mathfrak{N}_{1}=\ln \left(\int_{\mathbb{X}^{n}} \exp \left(\mathcal{N}_{1}\right) d Q_{2}\right)-\ln \left(\int_{\mathbb{X}^{n}} \exp \left(\mathcal{N}_{1}\right) d Q_{1}\right)=\sum_{j=0}^{d} \mathfrak{M}_{1 j}
$$

- сумма серии независимых одинаково распределенных случайных величин

$$
\mathfrak{M}_{1 j}=\ln \left(\int_{\mathbb{X}^{n}} \exp \left(\mathscr{M}_{1 j}\right) d Q_{2}\right)-\ln \left(\int_{\mathbb{X}^{n}} \exp \left(\mathscr{M}_{1 j}\right) d Q_{1}\right), \quad j=1, \ldots, d .
$$

Продолжим такой процесс «дробления» итеративным образом, беря за основу последовательно суммы $\mathscr{M}_{i}=\mathscr{M}_{i 1}=\sum_{t=1}^{n_{i}} \xi_{t}$ и соответствующие этим суммам статистики

$$
\begin{aligned}
\mathfrak{M}_{i}=\mathfrak{M}_{i 1}= & \ln \left(\int_{\mathbb{X}^{n}} \exp \left(\mathscr{M}_{i}\right) d Q_{2}\right) \\
& -\ln \left(\int_{\mathbb{X}^{n}} \exp \left(\mathscr{M}_{i}\right) d Q_{1}\right), \quad i=1, \ldots, r
\end{aligned}
$$

На $i$-м шаге $(i=1, \ldots, r)$ этого итеративного процесса полагаем $h=$ $h_{i}=n_{i}+m$ и разбиваем $\mathscr{M}_{i-1}$ на две части:

$$
\mathscr{M}_{i-1}=\sum_{j=0}^{d} \mathscr{M}_{i j}=\mathscr{N}_{i}+\mathscr{M}_{i 0}
$$

объединяя в основную часть $\mathscr{N}_{i}=\sum_{j=1}^{d} \mathscr{M}_{i j}$ независимые слагаемые

$$
\mathscr{M}_{i j}=\sum_{t=(j-1) h+1}^{(j-1) h+n_{i}} \xi_{t}, \quad j=1, \ldots, d
$$

В соответствии с этим разбиением статистика $\mathfrak{M}_{i-1}$ представляется в виде

$$
\mathfrak{M}_{i-1}=\sum_{j=0}^{d} \mathfrak{M}_{i j}=\mathfrak{N}_{i}+\mathfrak{M}_{i 0}
$$

где

$$
\mathfrak{N}_{i}=\ln \left(\int_{\mathbb{X}^{n}} \exp \left(\mathscr{N}_{i}\right) d Q_{2}\right)-\ln \left(\int_{\mathbb{X}^{n}} \exp \left(\mathscr{N}_{i}\right) d Q_{1}\right)=\sum_{j=1}^{d} \mathfrak{M}_{i j}
$$

сумма серии независимых одинаково распределенных случайных величин

$$
\mathfrak{M}_{i j}=\ln \left(\int_{\mathbb{X}^{n}} \exp \left(\mathscr{M}_{i j}\right) d Q_{2}\right)-\ln \left(\int_{\mathbb{X}^{n}} \exp \left(\mathscr{M}_{i j}\right) d Q_{1}\right),
$$


$j=1, \ldots, d$. Наряду с представлением (27) мы будем рассматривать представление

$$
\overline{\mathfrak{M}}_{i-1}=\sum_{j=0}^{d} \overline{\mathfrak{M}}_{i j}=\overline{\mathfrak{N}}_{i}+\overline{\mathfrak{M}}_{i 0}, \quad i=1, \ldots, r
$$

где

$$
\overline{\mathfrak{M}}_{i}=\mathfrak{M}_{i}-\mathbf{E} \mathfrak{M}_{i}, \quad \overline{\mathfrak{N}}_{i}=\mathfrak{N}_{i}-\mathbf{E} \mathfrak{N}_{i}, \quad \overline{\mathfrak{M}}_{i j}=\mathfrak{M}_{i j}-\mathbf{E} \mathfrak{M}_{i j}
$$

$j=0, \ldots, d, i=0, \ldots, r$.

3) В полученных представлениях (27) и (30) «остатки» $\mathfrak{M}_{i 0}$ и $\overline{\mathfrak{M}}_{i 0}$ достаточно малы:

$$
\left|\mathfrak{M}_{i 0}\right| \leqslant \tilde{\gamma}=C \gamma, \quad\left|\overline{\mathfrak{M}}_{i 0}\right| \leqslant \tilde{\gamma}, \quad \gamma \rightarrow 0 .
$$

Это обстоятельство позволяет свести оценки среднего и дисперсии статистики $\mathfrak{M}_{0}$ и (необходимого для проверки условия Ляпунова) четвертого момента статистики $\mathfrak{M}_{1}$ к оценкам моментов статистики $\mathfrak{M}_{r}$, для которой необходимые оценки получены в пп. 2.1 и 2.2 , ибо для $\mathfrak{M}_{r}$, согласно (22), $\delta n_{r} \rightarrow 0$.

Убедимся в справедливости (31). Из представлений (25)-(29) следует, что

$$
\begin{aligned}
\mathfrak{M}_{i 0}= & \ln \left(\frac{\int_{\mathbb{X}^{n}} \exp \left(\mathscr{N}_{i}+\mathscr{M}_{i 0}\right) d Q_{2}}{\int_{\mathbb{X}^{n}} \exp \left(\mathscr{N}_{i}+\mathscr{M}_{i 0}\right) d Q_{1}}\right)-\ln \left(\frac{\int_{\mathbb{X}^{n}} \exp \left(\mathscr{N}_{i}\right) d Q_{2}}{\int_{\mathbb{X}^{n}} \exp \left(\mathscr{N}_{i}\right) d Q_{1}}\right) \\
= & \ln \left(\frac{\int_{\mathbb{X}^{n}} \exp \left(\mathscr{N}_{i}\right) d Q_{2}+\int_{\mathbb{X}^{n}} \exp \left(\mathscr{N}_{i}\right)\left(\exp \left(\mathscr{M}_{i 0}\right)-1\right) d Q_{2}}{\int_{\mathbb{X}^{n}} \exp \left(\mathscr{N}_{i}\right) d Q_{1}+\int_{\mathbb{X}^{n}} \exp \left(\mathscr{N}_{i}\right)\left(\exp \left(\mathscr{M}_{i 0}\right)-1\right) d Q_{1}}\right) \\
& -\ln \left(\frac{\int_{\mathbb{X}^{n}} \exp \left(\mathscr{N}_{i}\right) d Q_{2}}{\int_{\mathbb{X}^{n}} \exp \left(\mathscr{N}_{i}\right) d Q_{1}}\right) \\
= & \ln \left(1+\frac{\int_{\mathbb{X}^{n}} \exp \left(\mathscr{N}_{i}\right)\left(\exp \left(\mathscr{M}_{i 0}\right)-1\right) d Q_{2}}{\int_{\mathbb{X}^{n}} \exp \left(\mathscr{N}_{i}\right) d Q_{2}}\right) \\
& -\ln \left(1+\frac{\int_{\mathbb{X}^{n}} \exp \left(\mathscr{N}_{i}\right)\left(\exp \left(\mathscr{M}_{i 0}\right)-1\right) d Q_{1}}{\int_{\mathbb{X}^{n}} \exp \left(\mathscr{N}_{i}\right) d Q_{1}}\right) .
\end{aligned}
$$

Легко видеть, что $d h_{i} \leqslant n_{i-1} \leqslant d h_{i}+d$, откуда следует, что сумма $\mathscr{M}_{i 0}$ состоит из не более чем $d m+d$ слагаемых из серии $\xi_{1}, \ldots, \xi_{n_{i-1}}$. Поэтому

$$
\left|\mathscr{M}_{i 0}\right| \leqslant C m d \delta
$$

и (ввиду условия $\gamma=\delta m d \rightarrow 0)\left|\exp \left(\mathscr{M}_{i 0}\right)-1\right| \leqslant C \mathscr{M}_{i 0} \leqslant \tilde{\gamma}$, что вместе с (32) и приводит к оценкам (31). 
2.4. Оценим среднее и дисперсию $\mathfrak{M}_{0}$ в случае $\delta n \geqslant C>0$ и $\delta m^{12} \rightarrow 0$.

1) Оценим среднее $a_{0}=\mathbf{E M}_{0}$. Положим

$$
a_{i}=\mathbf{E M}_{i}, \quad a_{i j}=\mathbf{E M}_{i j}, \quad j=0, \ldots, d, i=1, \ldots, r .
$$

Из (27) следует, что $a_{i-1}=d a_{i}+a_{i 0}, i=1, \ldots, r$, и потому

$$
a_{0}=d^{r} a_{r}+q
$$

где $q=a_{10}+d a_{20}+\cdots+d^{r-1} a_{r 0}$. Поскольку $\delta n_{r} \rightarrow 0$, то для $a_{r}$ можно применить полученную для случая $\delta n \rightarrow 0$ оценку (11), согласно которой

$$
a_{r} \sim-\frac{\delta^{2} n_{r}}{2} \quad \text { и } \quad d^{r} a_{r} \sim-\frac{\delta^{2} n}{2}
$$

Остается оценить $q$. Так как, согласно $(33), \exp \left(\mathscr{M}_{i 0}-1\right)=\mathscr{M}_{i 0}+O\left(\gamma^{2}\right)$, то из (32) следует, что

$$
\mathfrak{M}_{i 0}=W+O\left(\gamma^{2}\right)
$$

где

$$
W=\frac{\int_{X^{n}} \mathscr{M}_{i 0} \exp \left(\mathscr{N}_{i}\right) d Q_{2}}{\int_{\mathbb{X}^{n}} \exp \left(\mathscr{N}_{i}\right) d Q_{2}}-\frac{\int_{X^{n}} \mathscr{M}_{i 0} \exp \left(\mathscr{N}_{i}\right) d Q_{1}}{\int_{\mathbb{X}^{n}} \exp \left(\mathscr{N}_{i}\right) d Q_{1}}
$$

Далее,

$$
\begin{aligned}
|\mathbf{E} W| \leqslant \mid \mathbf{E}\left\{\left(\max _{x}\left\{\frac{\mathscr{M}_{i 0} \exp \left(\mathscr{N}_{i}\right)}{\exp \left(\mathscr{N}_{i}\right)}\right\}-\min _{x}\left\{\frac{\mathscr{M}_{i 0} \exp \left(\mathscr{N}_{i}\right)}{\exp \left(\mathscr{N}_{i}\right)} \mid X=x\right)\right\} \mid\right. \\
=\left|\mathbf{E}\left\{\left(\max _{x} \mathscr{M}_{i 0}-\min _{x} \mathscr{M}_{i 0}\right) \mid X=x\right\}\right| \leqslant C m d \delta^{2} \\
\left(x=\left\{x_{t}\right\}_{t=1}^{n} \in \mathbb{X}^{n}\right),
\end{aligned}
$$

так как $\mathscr{M}_{i 0}$ состоит из не более чем $(m+1) d$ слагаемых

$$
\xi_{t}=\ln \frac{p\left(Y_{t} \mid x_{t}\right)}{p_{1}\left(Y_{t}\right)}=\frac{p\left(Y_{t} \mid x_{t}\right)}{p_{1}\left(Y_{t}\right)}-1+O\left(\delta^{2}\right), \quad t=1, \ldots, n,
$$

и, при фиксированном $x_{t}$,

$$
\begin{aligned}
\mathbf{E}\left(\frac{p\left(Y_{t} \mid x_{t}\right)}{p_{1}\left(Y_{t}\right)}-1\right) & =\int_{\mathbb{Y}}\left(\frac{p\left(y_{t} \mid x_{t}\right)}{p_{1}\left(y_{t}\right)}-1\right) p\left(y_{t} \mid x_{t}\right) d \mu \\
& =\int_{\mathbb{Y}}\left(\frac{p\left(y_{t} \mid x_{t}\right)}{p_{1}\left(y_{t}\right)}-1\right)^{2} p_{1}\left(y_{t}\right) d \mu=O\left(\delta^{2}\right)
\end{aligned}
$$

В итоге имеем:

$$
\left|a_{i 0}\right| \leqslant C \gamma^{2} \quad \text { и } \quad q \leqslant C \gamma^{2} d^{r-1} .
$$


Собирая вместе оценки (34), (35), (36) и замечая, что, согласно (24), $q=o\left(\delta^{2} n\right)$, мы приходим к оценке $a_{0} \sim-\delta^{2} n / 2$.

2) Оценим дисперсию $b_{0}^{2}=\mathbf{D} \mathbb{M}_{0}$, для чего положим

$$
\alpha=\sqrt{d}, \quad b_{i}^{2}=\mathbf{D M}_{i}, \quad\|\xi\|=\sqrt{\mathbf{E} \xi^{2}}
$$

и воспользуемся неравенством треугольника $\left\|\overline{\mathfrak{N}}_{i}\right\|-\left\|\overline{\mathfrak{M}}_{i 0}\right\| \leqslant\left\|\overline{\mathfrak{M}}_{i-1}\right\| \leqslant$ $\left\|\overline{\mathfrak{N}}_{i}\right\|+\left\|\overline{\mathfrak{M}}_{i 0}\right\|$, из которого следует, что

$$
\alpha b_{i}-\tilde{\gamma} \leqslant b_{i-1} \leqslant \alpha b_{i}+\tilde{\gamma}, \quad i=1, \ldots, r,
$$

и

$$
\alpha^{r} b_{r}-\tilde{\gamma}\left(1+\alpha+\cdots+\alpha^{r-1}\right) \leqslant b_{0} \leqslant \alpha^{r} b_{r}-\tilde{\gamma}\left(1+\alpha+\cdots+\alpha^{r-1}\right) .
$$

В п. 2.1 доказано, что

$$
b_{r}^{2} \sim \delta^{2} n_{r} \sim \Delta^{r} \delta^{2} n
$$

и это вместе с (37) приводит к оценке

$$
\left|b_{0}-\delta \sqrt{n}\right| \leqslant C \gamma \frac{\alpha^{r}-1}{\alpha-1} \leqslant C \gamma \alpha^{r-1}
$$

Остается заметить, что правая часть в этом неравенстве, согласно (24), по порядку меньше, чем $\delta \sqrt{n}$ :

$$
\frac{\gamma \alpha^{r-1}}{\delta \sqrt{n}}=\frac{\delta m d \alpha^{r-1}}{\delta \sqrt{n}}=\frac{m}{\sqrt{\Delta^{r+1} n}}=o(1) .
$$

2.5. Покажем асимптотическую нормальность $\widetilde{L}_{n}=\overline{\mathfrak{M}}_{0} / b_{0}$ в случае $\delta n \geqslant C>0$ и $\delta m^{12} \rightarrow 0$.

Из (38) и (39) следует, что предельное распределение статистики $\overline{\mathfrak{M}}_{0}$ совпадает с предельным распределением суммы $d^{r}$ в независимых случайных величин, четвертые моменты которых, согласно (16), ограничены величиной $C \delta^{4} n_{r}^{2}$. Таким образом, выполнение для данной суммы условия Ляпунова $d^{r} \delta^{4} n_{r}^{2}=o\left(\left(\mathbf{D} \overline{\mathfrak{M}}_{0}\right)^{2}\right)$ обеспечивает асимптотическую нормальность статистики $\overline{\mathfrak{M}}_{0} / b_{0}$.

2.6. Заметим, что в случае выполнения условий (6) для доказательства асимптотической нормальности $\widetilde{L}_{n}$ достаточно в описанной (в пп. 2.3 и 2.4) процедуре дробления положить $r=4$. При этом, так как $\delta^{2} n=$ const $(>0)$, из асимптотической нормальности ЛОП при гипотезе $H_{1}$ с параметрами $\left(-\delta^{2} n / 2, \delta^{2} n\right)$ вытекает контигуальность гипотез $H_{1}$ и $H_{2}$ относительно распределения последовательности $Y$. Воспользовавшись свойствами контигуальных гипотез (см. $[10$, с. 43$])$, получаем, что 
при условии гипотезы $H_{2}$ статистика $L_{n}$ асимптотически нормальна с параметрами $\left(-\delta^{2} n / 2, \delta^{2} n\right)$.

В общем случае доказательство теоремы при условии гипотезы $H_{2}$ заключается в том, чтобы повторить рассуждения, проведенные для доказательства теоремы ; условиях гипотезы $H_{1}$. Структура доказательства при этом сохранится, а изменения произойдут только в некоторых численных выражениях.

\section{СПИСОК ЛИТЕРАТУРЫ}

1. Ибрагимов И. А., Линиик Ю. В. Независимые и стационарно связанные величины. М.: Наука, 1965, 524 с.

2. Hoeffding $W$., Robbins $H$. The central limit theorem for dependent random variables. - Duke Math. J., 1948, v. 15, p. 773-780.

3. Diananda $P$. $H$. The central limit theorem for $m$-dependent variables. - Proc. Cambridge Philos. Soc., 1955, v. 51, p. 92-95.

4. Berk $K$. $N$. A central limit theorem for $m$-dependent random variables with unbounded $m$. - Ann. Probab., 1973, v. 1, p. 352-354.

5. Розанов Ю. А. Стационарные случайные процессы. М.: Наука, 1990, 271 с.

6. Грияь $A . \Gamma$. Гредельные теоремы для схем серий слабо зависимых величин. Теория вероятн. и ее примен., 1995, т. 40, в. 4, с. 888-897.

7. Малевчч T. Л., Абдалимов Б. Уточнение центральной предельной теоремы для $U$-статистик от $m$-зависимых величин. - Теория вероятн. и ее примен., 1982, т. XXVII, в. 2, с. 369-373.

8. Проскурия $Г$. В. Задача различения гипотез о параметрах процесса обобщенного скользящего суммиров гния. - Дискретн. матем., 1993, т. 5, в. 3, с. 44-63.

9. Arbekov I. M. Asymptotically optimum detection of a weak signal sequence with random time delays. - IEEE Trans. Inform. Theory, 1995, v. 41, № 4, p. 1169-1174.

10. Русас Дж. Контигуальность вероятностных мер. М.: Мир, 1975, 254 с.

11. Ибрагимов И. А., Хасьминский $Р$. З. Асимптотическая теория оценивания. М.: Наука, 1979, $527 \mathrm{c}$.

12. Линъков Ю. Н. Асимптотические методы статистики случайных процессов. Киев: Наукова думка, 1993, 255 с.

13. Тараския $A$. Ф. Асимптотическое поведение отношения правдоподобия семимартингалов. - В сб.: Статистика и управление случайными процессами. М.: Наука, 1989 , c. $199-202$.

14. Жакод Ж., ШІиряев А. Н. Предельные теоремы для случайных процессов. Т. 2. М.: Физ.-мат. лит., 1994, 367 с.

15. Bernstein $S$. N. Sur l'extention du théorème limite du calcul des probabilités aux sommes de quantités dependantes. -- Math. Ann., 1926, v. 97, p. 1-59. (Бернитейя C. H. Распространение предельной теоремы теори и вероятностей на суммы зависимых величин. - Успехи матем. наук, 1944, т. 10, с. 65-114.)

16. Oosterhoff J., Van Zwet W. R. A note on contiguity and Hellinger distance. - In: Contributions to Statistics. Ed. by J. Jureckova. Prague: Academia, 1979, p. 157-166. 